\title{
Nuclear analyses for the design of the ITER-Like Plasma Facing Components vertical targets of the DEMO Divertor
}

\author{
Simone Noce ${ }^{\mathrm{a}}$, Giacomo Dose ${ }^{\mathrm{a}}$, Davide Flammini ${ }^{\mathrm{b}}$, Vito Imbriani $^{\mathrm{c}}$, Giuseppe Mazzone ${ }^{\mathrm{b}}$, Fabio Moro $^{\mathrm{b}}$, \\ Selanna Roccella ${ }^{\mathrm{b}}$, Francesco Romanelli ${ }^{\mathrm{a}}$, Rosaria Villari ${ }^{\mathrm{b}}$, Eliseo Visca ${ }^{\mathrm{b}}$, Jeong-Ha You ${ }^{\mathrm{d}}$ \\ ${ }^{a}$ University of Rome Tor Vergata, Industrial Engineering Department, via del Politecnico 1, 00133, Rome, Italy \\ ${ }^{b}$ ENEA FSN-FUSTEC-TEN, C.R. Frascati, via E. Fermi 45, 00044 Frascati (Rome), Italy \\ ${ }^{c}$ CREATE/University of Naples, Industrial Engineering, Piazzale Tecchio 80, 80125 Napoli, Italy \\ ${ }^{d}$ Max-Planck-Institut für Plasmaphysik, Boltzmannstrasse 2, 85748 Garching, Germany
}

\begin{abstract}
The Divertor Plasma Facing Components (PFCs) of a fusion reactor are the most loaded components in terms of high heat fluxes, which, combined with high neutron irradiation, can severely compromise their thermo-mechanical and physical properties as well as their heat removal capacity. Therefore, neutronic assessment plays a key role in the design of these critical components. The aim of this work is to perform a dedicated nuclear analysis for the European DEMO divertor PFCs placed on the vertical targets, aimed to provide significant outcomes in the PFCs selection concept. In particular, the present assessment is devoted to the reference ITER-like configuration under study within the EUROfusion WPDIV-PPPT programme. Three-dimensional neutronics analyses have been performed with the MCNP5 Monte Carlo code. This work presents detailed neutronics results with heterogeneous materials constitution and actual geometry of the PFC concept. High resolution data on the nuclear heating density and neutron damage of the ITER-like PFCs placed on the divertor vertical targets, including helium production, assessed for first time for the latest DEMO design, are presented and discussed.
\end{abstract}

Keywords: PFC, DEMO, neutronics, Divertor, MCNP

\section{Introduction}

One of the most critical missions of the recent European roadmap drafted for realizing commercially viable fusion power generation, is the reliable power handling [1,2]. In this regard, the divertor is the key in-vessel component, as it is responsible for power exhaust and impurity removal via guided plasma exhaust [3]. Due to the intense bombardment of energetic plasma particles, the Plasma-Facing Components (PFCs) of the divertor are exposed to extreme heat flux loads. In addition, neutron irradiation produces defects and damage in the materials leading to embrittlement, hardening, reduced thermal conductivity and creep. These effects normally exhibit significant temperature-dependence and thus are likely to be affected by operation conditions [4]. Therefore, the evaluation of nuclear loads due to neutrons and secondary gamma on the divertor targets, is fundamental in the design of these critical components.

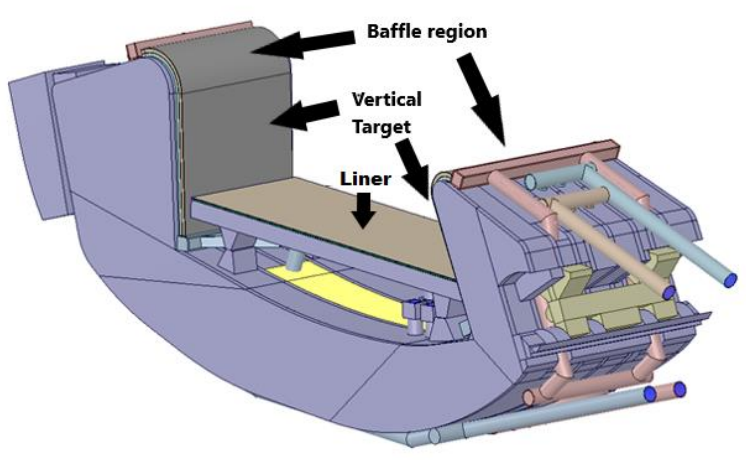

Fig. 1. Latest neutronics CAD model of the DEMO divertor cassette [5].
The PFC of a divertor cassette consists of three parts, namely, vertical target, liner (replaced by the dome in some configurations) and baffle [4]. The 2018 DEMO divertor cassette layout is shown in figure 1 [5]. In this work, specific neutronics analyses have been performed on the straight leg of Inner and Outer Vertical Targets (IVT and OVT). These components have been modelled with a high level of detail, following the actual geometry and materials description of the main PFC concept of interest for DEMO: the reference ITER-like (IL) design [6]. For the neutronics modelling of the baffle and liner region it has been assumed that the section of the PFCs consists of 3 homogenized layers, representing the actual volume fraction of constituent materials in the PFC. Nuclear heating density, nuclear damage and helium production distributions have been calculated in each subcomponent of the IL PFCs, both for IVT and for OVT through neutron and gamma transport simulations using MCNP5v1.6 Monte Carlo code [7] and the Joint Evaluated Fusion File JEFF 3.3 nuclear data libraries [8]. The MCNP reference damage cross-sections model is based on the NRT method [9]. The estimation of the nuclear loads provides data for the thermo-mechanical and safety analyses and allows to obtain important information for the design, lifetime and operations of these components.

\section{ITER-like concept: design and MCNP modelling}

The design of the ITER-like concept basic unit and the equivalent MCNP model are shown in figure 2 and 3 respectively. Each IL monoblock is made of tungsten and it's $28 \mathrm{~mm}$ high, $23 \mathrm{~mm}$ wide and $12 \mathrm{~mm}$ thick. It is 
crossed by a $\mathrm{CuCrZr}$ cooling pipe with internal and external diameter of 12 and $15 \mathrm{~mm}$ respectively. These two components are separated by $1 \mathrm{~mm}$ thick pure copper interlayer. The minimum distance between the interlayer outer surface and the three side walls of the monoblock is $3 \mathrm{~mm}$ while the distance from the monoblock upper surface is $8 \mathrm{~mm}$.

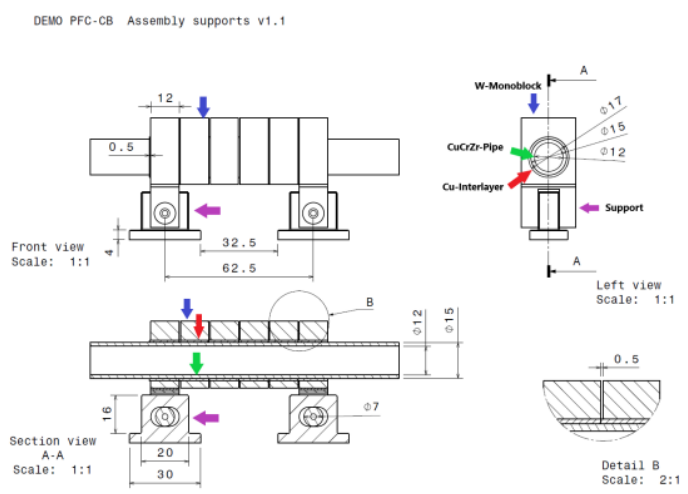

Fig. 2. Geometrical description of the ITER-like PFC concept [6].

The supports have been modeled in details as shown in figure 2 and 3. The pre-processing and preparation of the IL CAD model has been performed by means of the $3 \mathrm{D}$ modeling software Ansys SpaceClaim 2019 [10] in order to generate a CAD model suitable for neutronic analyses. The simplified CAD model has been converted into the equivalent MCNP geometrical representation using the CAD-to-MCNP interface of SuperMC [11].

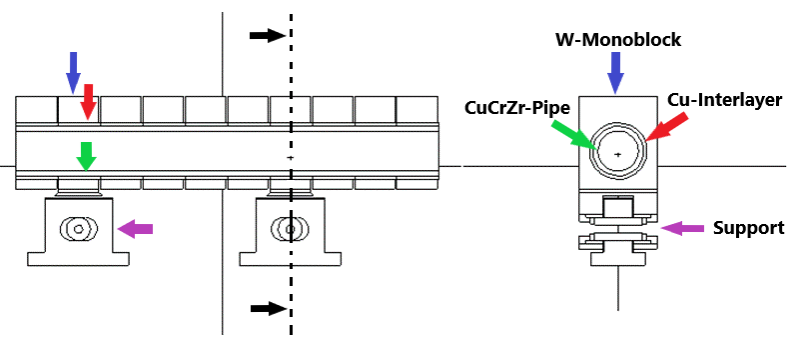

Fig. 3. MCNP model of a basic unit of the ITER-like PFC concept.

The detailed MCNP model of the PFC (figure 3) consists of a basic unit including ten $\mathrm{W}$-mononblocks and $\mathrm{Cu}$ interlayers, two supports and one $\mathrm{CuCrZr}$-pipe segment $12.5 \mathrm{~cm}$ long. It has been manually integrated and replicated into the IVT and OVT straight leg of the divertor heterogeneous model, developed for 2018 neutronics study [12]. The total number of monoblocks and interlayers arranged on the IVT and OVT is shown in table 1 and figure 4 . Vertically, the CuCrZr-pipes are subdivided into 6 and 5 continuous and equal segments, respectively on IVT and OVT (table 1 and figure 4). The neutronics model of the baffle regions PFC consists of 3 homogenized layers, representing the actual volume fraction of constituent materials: the outermost layers are composed of tungsten (W: 94 vol.\%) and void (6 vol.\%) to take into account the gaps $(0.05 \mathrm{~cm})$ between the monoblocks, the middle layer is a mixture of tungsten (W: 39.41 vol.\%), water (28.31 vol.\%), CuCrZr (15.92 vol.\%) and void (16.36 vol.\%). The DEMO reactor layout used in the present studies is based on EU
DEMO1 2017 reference configuration model representing a $11.25^{\circ}$ toroidal sector of the tokamak [13], with the WCLL SMS Blanket (figure 5) and with plasma parameters shown in table 2. It has a quasiheterogeneous blanket representation and heterogeneous divertor. The WCLL Breeding Blanket model is based on layered configuration along the radial direction [14] representing the internal blanket structure: first wall and $\mathrm{W}$-armor, breeding zone and manifolds. The materials properties and composition have been selected according to the specifications for neutronic analyses in the EUROfusion framework [15].

Table 1. Vertical and horizontal arrangement of Monoblocks, Interlayers and $\mathrm{CuCrZr}$-pipes in the MCNP model.

\begin{tabular}{l|l|l}
\hline & IVT & OVT \\
\hline Number of vertical Monoblocks and Interlayers & 57 & 50 \\
\hline $\begin{array}{l}\text { Number of horizontal Monoblocks, Interlayers and } \\
\text { CuCrZr pipes }\end{array}$ & 31 & 43 \\
\hline Number of vertical CuCrZr-pipe segments & 6 & 5 \\
\hline
\end{tabular}
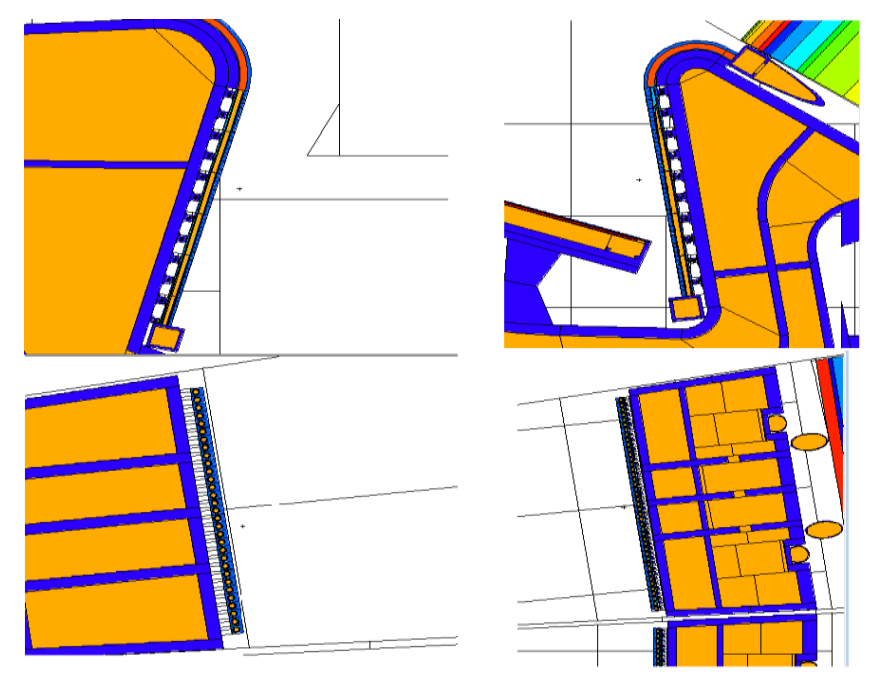

Fig. 4. DEMO divertor PFCs target MCNP model: poloidal section (top) and toroidal section (bottom) showing the Inner (left) and Outer (right) Vertical Target.

Table 2: Main parameters of the DEMO baseline configuration.

\begin{tabular}{l|c}
\hline $\mathrm{N}^{\circ}$ of Toroidal Field Coils & 16 \\
Major radius $(\mathrm{m})$ & 8.938 \\
Minor radius $(\mathrm{m})$ & 2.883 \\
Aspect ratio & 3.1 \\
Plasma elongation & 1.65 \\
Plasma triangularity & 0.33 \\
Fusion power & 1998 \\
Average neutron wall loading $\left(\mathrm{MW} / \mathrm{m}^{2}\right)$ & 1.04 \\
Net electric power & 500 \\
\hline
\end{tabular}

\section{Nuclear analyses}

The developed MCNP DEMO model with 2018 divertor and PFCs vertical target has been used to assess the nuclear loads on these components (IL) and to verify which are the most critical and loaded parts of the IVT and OVT. The nuclear heating, neutron damage and Heproduction distribution have been evaluated using fine 2D-meshes, with the following dimensions: $57 \times 31$ is the IVT resolution, 50x43 is the OVT resolution (number of 
vertical and horizontal W-monoblocks and $\mathrm{Cu}$ interlayers, respectively). The volume of each $\mathrm{W}$ monoblock and $\mathrm{Cu}$-interlayer is around 5 and $0.6 \mathrm{~cm}^{3}$ respectively. The nuclear loads on $\mathrm{CuCrZr}$-pipes have been plotted on graphs, showing the loads distribution as a function of the horizontal number of pipes and the number of vertical segments. The results have been normalized to $1998 \mathrm{MW}$ fusion power (neutron yield: $7.094 \times 10^{20} \mathrm{n} / \mathrm{s}$ ), according to the plasma parameters specified in table 2 .

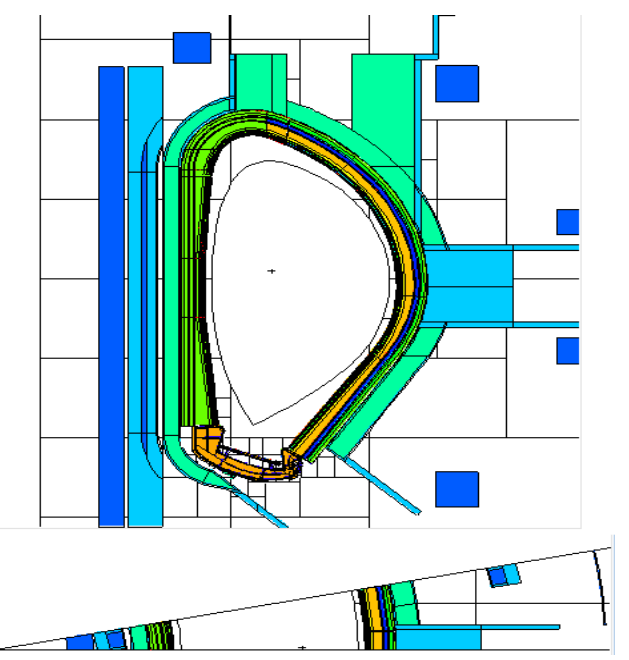

Fig. 5. DEMO MCNP model with SMS quasi-heterogeneous WCLL blanket [14] and divertor [13]: poloidal section showing the Inboard and Outboard breeding blanket and divertor (top) and toroidal section along the equatorial plane (bottom).

The simulations have been performed using standard MCNP cell-based tallies (F4) with proper multiplier to calculate the nuclear quantities of interest for the PFCs design development, performances assessment and verification of design requirements [12]. The results have statistical uncertainties in the range $\pm 0.5 \%-5 \%$ (depending on the position and dimensions of the cell under analysis) with a maximum of $\pm 7 \%$ in few limited zones. The calculations have been performed on the ENEA CRESCO cluster [16].

\subsection{Nuclear heating density, damage and He- production distribution on IVT and OVT}

The nuclear heating density distributions on IVT and OVT for the $\mathrm{W}$ monoblocks of the IL PFC concept are shown in figure 6 . For both vertical targets the nuclear heating peak values are on the monoblocks closest to the baffle region (approximately $20 \mathrm{~W} / \mathrm{cm}^{3}$ in OVT and 19 $\mathrm{W} / \mathrm{cm}^{3}$ in IVT), then decreasing along the vertical direction (less than $10 \mathrm{~W} / \mathrm{cm}^{3}$ at the bottom section). Furthermore, the nuclear heating values are generally greater at the external edges of the targets, decreasing gradually towards the internal monoblocks, highlighting a symmetry with respect to the central axis of the targets. This means that the most loaded parts of the targets are the monoblocks near the baffle region and those placed on the external edges, i.e. close to the gap between the cassette. If a value of $10 \mathrm{~W} / \mathrm{cm}^{3}$ is considered, $93 \%$ of the IVT monoblocks are subject to a nuclear heating density greater than $10 \mathrm{~W} / \mathrm{cm}^{3}$. This percentage is lower for the OVT (about $88 \%$ ). This means that there is a greater portion of $\mathrm{W}$-monoblocks more stressed in terms of neutronics thermal load on the IVT than the OVT. This is due to the different plasma field of view factor of the two targets, which appears to be more extended for the inner vertical target. The nuclear heating density distributions on IVT and OVT for the $\mathrm{Cu}$-interlayers are shown in figure 7. The distributions are very similar to the W-monoblocks case: for both vertical targets, the nuclear heating peak values are on the interlayers closest to the baffle region (around $7.5 \mathrm{~W} / \mathrm{cm}^{3}$ in OVT and 7 $\mathrm{W} / \mathrm{cm}^{3}$ in IVT), then they decrease along the vertical direction (less than $3 \mathrm{~W} / \mathrm{cm}^{3}$ at the bottom section). As in the previous case, the nuclear heating values are generally greater at the external edges of the targets, with a gradually reduction along the more internal interlayers, showing the same kind of symmetry observed for the Wmonoblocks. Considering a reference value of $4 \mathrm{~W} / \mathrm{cm}^{3}$, a greater portion of interlayers results to be more stressed $\left(>4 \mathrm{~W} / \mathrm{cm}^{3}\right)$ from the point of view of neutronics thermal load, on the IVT (79\%) than the OVT $(60 \%)$. The nuclear heating density distributions on IVT and OVT for the CuCrZr-pipes are shown in figure 8. In this case, the pipe has been subdivided along the vertical direction into 5 and 6 continuous segments of equal length, respectively for OVT and IVT. These graphs highlight a trend of the nuclear heating distribution on CuCrZr-pipes already evidenced with the previous meshes: in both cases the nuclear heating peak values are on the upper parts of the pipe close to the external edges of the targets (slightly less than $7 \mathrm{~W} / \mathrm{cm}^{3}$ ), then decreasing along the vertical direction (around $3 \mathrm{~W} / \mathrm{cm}^{3}$ at the bottom section).
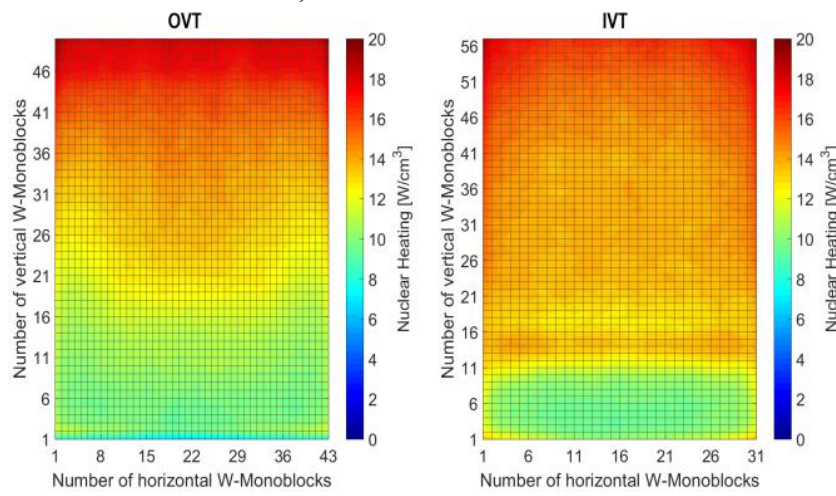

Fig. 6. Nuclear heating density distribution on W-Monoblocks OVT (left) and IVT (right).
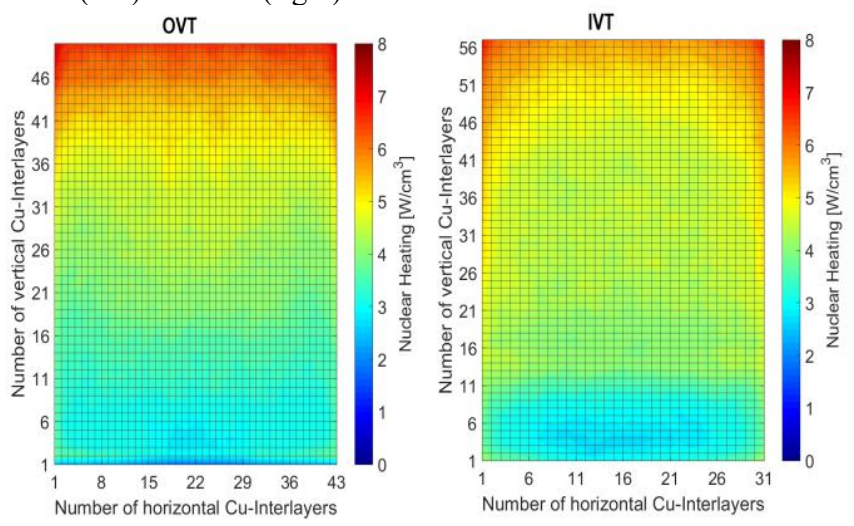

Fig. 7. Nuclear heating density distribution on $\mathrm{Cu}$-Interlayers OVT (left) and IVT (right). 
As in the previous cases, a symmetrical behavior of the nuclear heating distribution with respect to the axis passing through the center of the targets is observed.

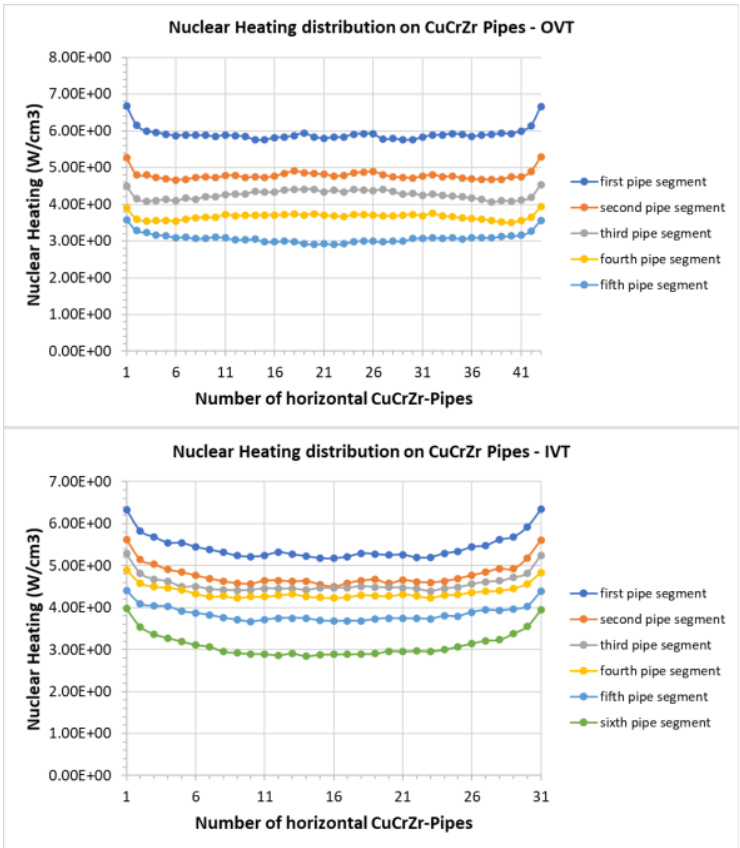

Fig. 8. Nuclear heating density distribution on $\mathrm{CuCrZr}$-pipes OVT (top) and IVT (bottom) as function of the CuCrZr-pipes horizontal number for each vertical pipe segments, starting from the upper part (first) to the bottom part (fifth or sixth).

The nuclear damage distributions on IVT and OVT for the $\mathrm{W}$-monoblocks are shown in figure 9. The damage peak value is located in the upper part and external layers of the targets: about $1.3 \mathrm{dpa} / \mathrm{FPY}$ in OVT and 1.37 $\mathrm{dpa} / \mathrm{FPY}$ in IVT. There is a gradual decrease along the vertical direction, reaching a value lower than 0.3 dpa/FPY in the bottom section of both the targets (minimum: $0.2 \mathrm{dpa} / \mathrm{FPY}$ on the OVT). Also for the damage, a symmetrical distribution with respect to the central axis of the target is found. If a reference value $0.75 \mathrm{dpa} / \mathrm{FPY}$ is considered, about $53 \%$ of these components are subjected to a damage greater than the reference, compared to the OVT (29\%). The nuclear damage distributions on IVT and OVT for the $\mathrm{Cu}$ interlayers are shown in figure 10. The damage peak value is in the upper part and external borders of the targets: about $5.70 \mathrm{dpa} / \mathrm{FPY}$ for the IVT and $6 \mathrm{dpa} / \mathrm{FPY}$ for the OVT. There is a gradual reduction along the vertical direction up to values lower than $1 \mathrm{dpa} / \mathrm{FPY}$ in the bottom section of both the targets.

The symmetrical distribution behavior with respect to the target center axis is found also for this subcomponent and $43 \%$ of the IVT interlayers show a damage level greater than $3 \mathrm{dpa} / \mathrm{FPY}$; this percentage decreases to $23 \%$ on the OVT. The nuclear damage distributions on IVT and OVT for the $\mathrm{CuCrZr}$ pipes are shown in figure 11. The damage peak value is on the upper part and external borders of the targets: about $4.80 \mathrm{dpa} / \mathrm{FPY}$ for the IVT and $4.77 \mathrm{dpa} / \mathrm{FPY}$ for the OVT. There is a gradual reduction along the vertical direction up to values a bit higher than $1 \mathrm{dpa} / \mathrm{FPY}$ in the bottom section of both the targets (minimum: $1.2 \mathrm{dpa} / \mathrm{FPY}$ on the IVT). It should be noted that the maximum nuclear loads of the
PFC are not in the target but in the baffle regions, represented with three homogeneous layers in the present model. In particular for the present design the nuclear damage in $\mathrm{W}, \mathrm{Cu} / \mathrm{CuCrZr}$ reach the maximum values of about $2 \mathrm{dpa} / \mathrm{FPY}$ and $7 \mathrm{dpa} / \mathrm{FPY}$, respectively in the baffle zone (about $40 \%$ higher than in the target) [12].
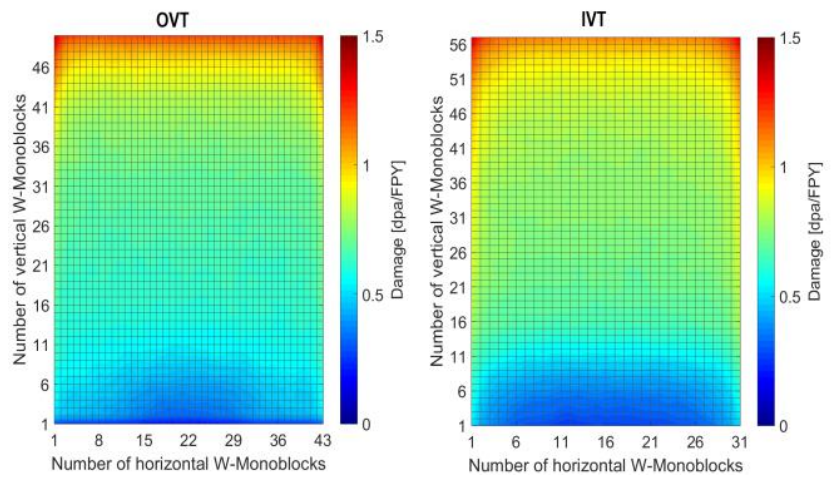

Fig. 9. Nuclear damage distribution on W-Monoblocks OVT (left) and IVT (right).
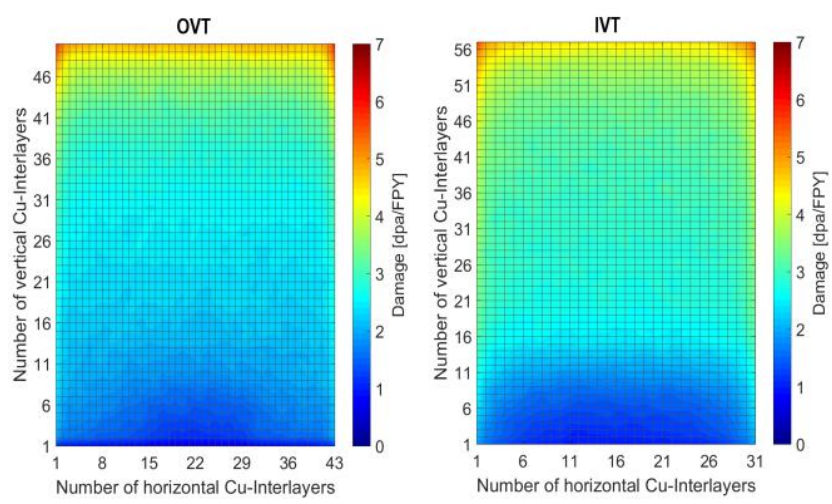

Fig. 10. Nuclear damage distribution on $\mathrm{Cu}$-Interlayers OVT (left) and IVT (right).

Taking into account the previous results, the maximum cumulated damage in the $\mathrm{W}$ armor, integrated over 2 FPY (specified PFC and divertor lifetime), reach 4 dpa on the baffle region. This peak value decreases up to 2.9 dpa if the straight leg of the vertical targets is considered. The minimum cumulated damage on $\mathrm{W}$ is reached in the central and bottom part of the outer vertical target (about $0.4 \mathrm{dpa}$ ), highlighting a range of variation between 0.4 and $4 \mathrm{dpa}$. In the $\mathrm{CuCrZr}$ tube, the maximum integrated damage is about $14 \mathrm{dpa}$, on the baffle region, decreasing up to $9.6 \mathrm{dpa}$ on the upper and external part of the straight leg of the vertical targets. The minimum is in the lower and central sections of the IVT (2.4 dpa), underlining a variation range of 2.4-14 dpa. According to the previous irradiation test data, $\mathrm{CuCrZr}$ exhibits saturation of tensile behavior in the dose range of 0.5-2.5 dpa [17] or 1-10 dpa [18] at 150$300^{\circ} \mathrm{C}$. Thus, the peak dpa values (10-14 dpa) may probably be acceptable [19]. Furthermore, it can be noted that the maximum values for all the assessed nuclear responses in the targets are localized in the OVT. This is confirmed also by the He-production distributions on IVT and OVT for the W-monoblocks and $\mathrm{Cu}$-Interlayers, which are shown in figures 12 and 13. 


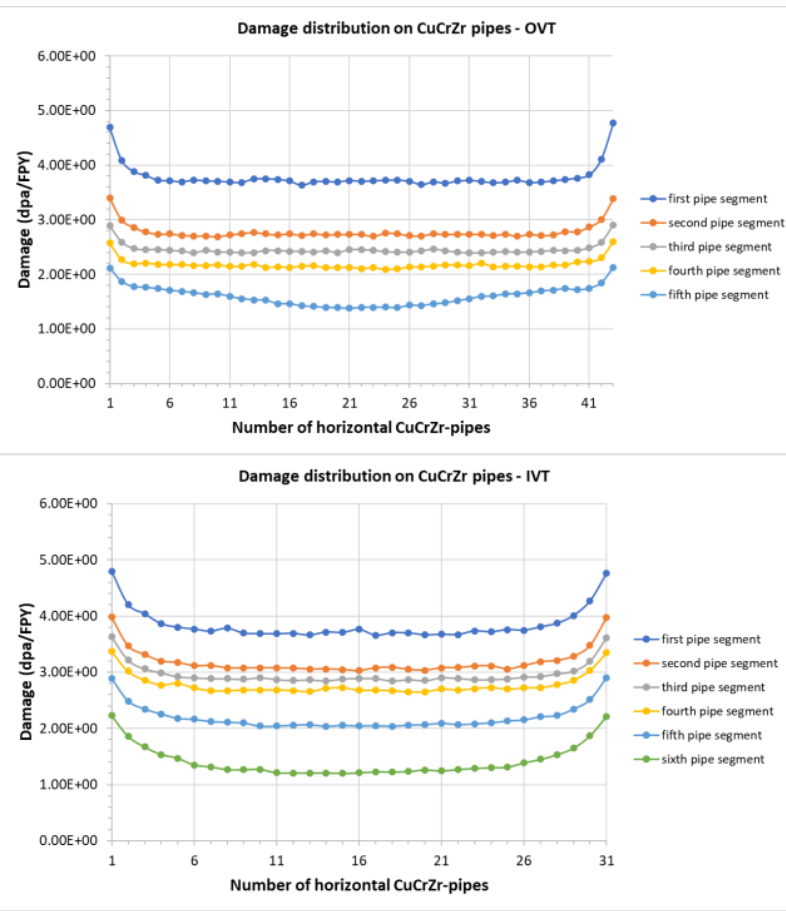

Fig. 11. Nuclear damage distribution on $\mathrm{CuCrZr}$-pipes OVT (top) and IVT (bottom) as function of the CuCrZr-pipes horizontal number for each vertical pipe segments, starting from the upper part (first) to the bottom part (fifth or sixth).
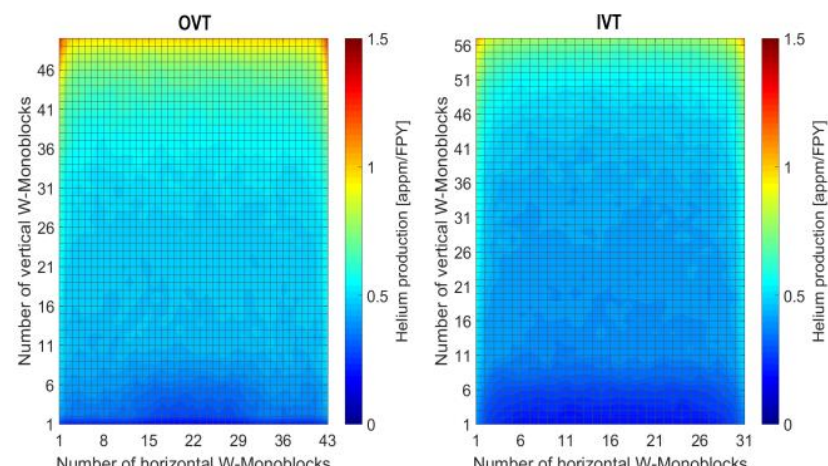

Fig. 12. Helium production distribution on W-Monoblocks OVT (left) and IVT (right).

For both the components, the He-production peak value is in correspondence of the upper part and external edges of the targets: about 1.1 appm/FPY (IVT) and 1.3 appm/FPY (OVT) on tungsten, $31 \mathrm{appm} / \mathrm{FPY}$ (IVT) and $39 \mathrm{appm} / \mathrm{FPY}$ (OVT) on copper. The reduction along the vertical direction and the symmetrical behavior of the distribution respect to the target center are confirmed. At the bottom section of the targets the He-production values drop below 0.2 appm/FPY (W-monoblocks) and 4 appm/FPY (Cu-interlayers). The He-production distributions on IVT and OVT for the CuCrZr-pipes are shown in figure 13. The peak values are: $25 \mathrm{appm} / \mathrm{FPY}$ (IVT) and $30 \mathrm{appm} / \mathrm{FPY}$ (OVT), located in the external edges of the targets near the baffle region. The minimum He-production is in the bottom part and central section (target symmetry): $5.6 \mathrm{appm} / \mathrm{FPY}$ (IVT) and 7.6 appm/FPY (OVT). Therefore, at the end of the DEMO divertor lifetime (2 FPY) the following cumulative quantities of Helium produced on the PFCs vertical targets are expected: $0.4-2.6$ appm on $\mathrm{W}$-monoblocks,
8- 78 appm on $\mathrm{Cu}$-interlayers, $11-60$ appm on $\mathrm{CuCrZr}$ heat sink material.
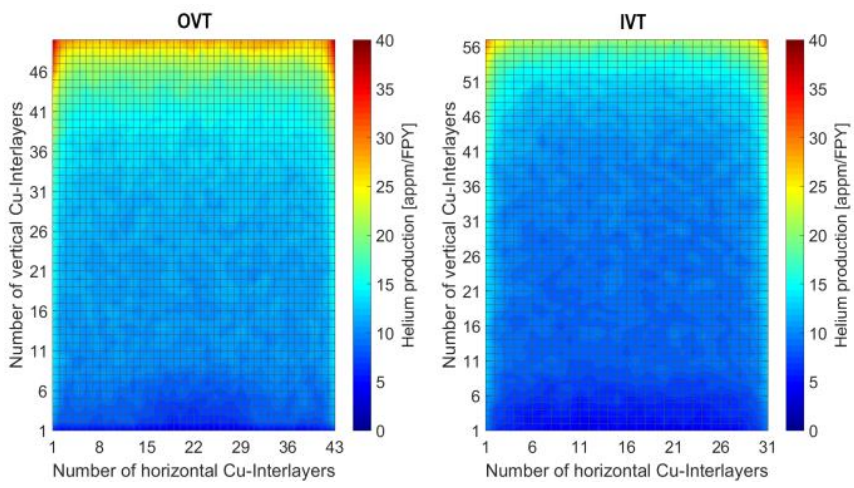

Fig. 13. Helium production distribution on $\mathrm{Cu}$-Interlayers OVT (left) and IVT (right).

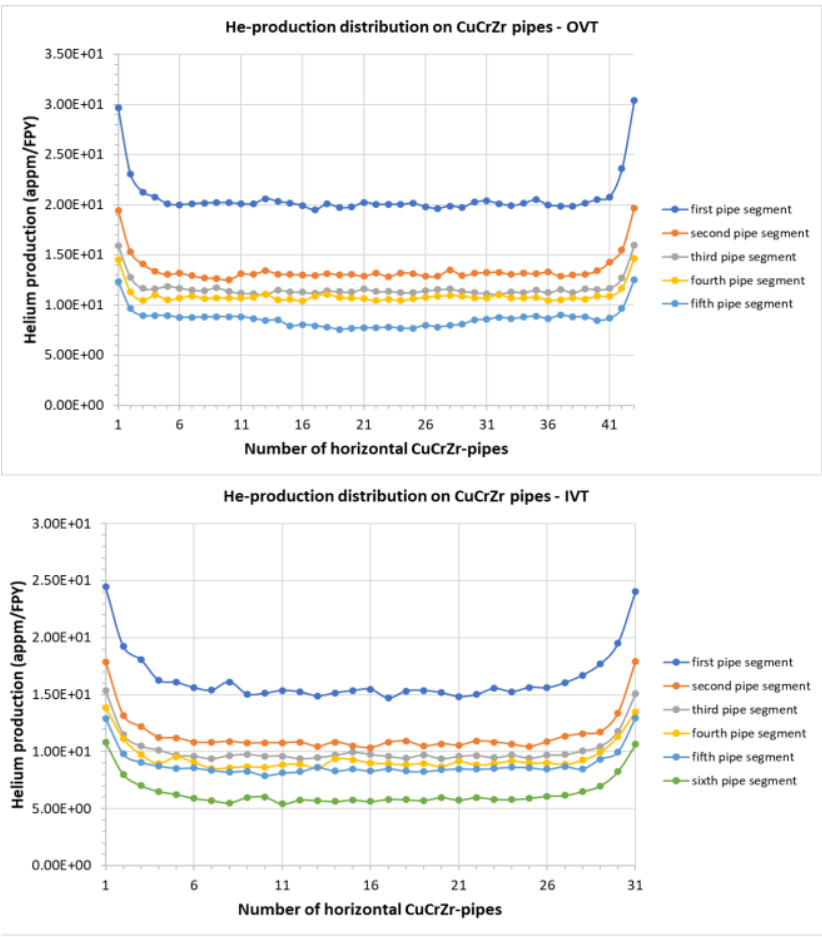

Fig. 14. Helium production distribution on CuCrZr-pipes OVT (top) and IVT (bottom) as function of the CuCrZr-pipes horizontal number for each vertical pipe segments, starting from the upper part (first) to the bottom part (fifth or sixth).

\section{Conclusions}

These analyses have been carried out on the most reliable candidate for the DEMO divertor PFC concepts: ITER-like design. A detailed MCNP model of the straight legs of the PFC vertical targets has been generated and used to estimate the nuclear loads for the IL vertical targets. All the quantities show peak values at the top and on the outer edges of the targets (near the baffle region and a gradual decrease along the vertical direction (in particular towards the center of the target) highlighting a symmetrical distribution with respect to the target central axis. Furthermore, it has been shown that, despite of the fact that the maximum values are generally located in OVT, the IVT is subject to a more extended irradiation level, as seen for the nuclear heating and nuclear damage, due to a broader field of view of the 
plasma. In conclusion, at the end of the of the divertor lifetime (2 FPY) the following cumulative ranges of variation in terms of dpa and He-production in the vertical targets are expected: $0.4-2.9$ dpa on Wmonoblocks (maximum on the baffle region: $4 \mathrm{dpa}$ ), 1.2 - 12 dpa on Cu-interlayers, $2.4-9.6$ dpa on $\mathrm{CuCrZr}$ pipes (maximum on the baffle region: $14 \mathrm{dpa}$ ); $0.4-2.6$ appm on $\mathrm{W}$-monoblocks, 8 - 78 appm on $\mathrm{Cu}$-interlayers, $11-60$ appm on $\mathrm{CuCrZr}$-pipes.

\section{Acknowledgments}

This work has been carried out within the framework of the EUROfusion Consortium and has received funding from the Euratom research and training programme 2014-2018 and 2019-2020 under grant agreement No 633053. The views and opinions expressed herein do not necessarily reflect those of the European Commission.

The computing resources and the related technical support used for this work have been provided by CRESCO/ENEAGRID High Performance Computing infrastructure and its staff [15]. CRESCO/ENEAGRID High Performance Computing infrastructure is funded by ENEA, the Italian National Agency for New Technologies, Energy and Sustainable Economic Development and by Italian and European research programmes, see http://www.cresco.enea.it/english for information.

\section{References}

[1] T. Donné et al., European Research Roadmap to the Realisation of Fusion Energy, ISBN 978-3-00-061152-0, November 2018.

[2] G. Federici et al., Overview of the DEMO staged design approach in Europe, Nucl. Fusion 59 (2019) 066013.

[3] J.H. You et al., Conceptual design studies for the European DEMO divertor: Rationale and first results, Fusion Engineering and Design 109-111 (2016) 1598-1603.

[4] J.H. You, A review on two previous divertor target concepts for DEMO: mutual impact between structural design requirements and materials performance, Nucl. Fusion 55 (2015) 113026.

[5] D. Marzullo, https://idm.euro-fusion.org/?uid=2N7KXG DEMO_Divertor_2018_v2.0 (2N7KXG)

[6] F4E-OMF-567 - Annex B - Technical Specification (F4E_D_27MKGH v.1.5)

[7] X-5 Monte Carlo Team: MCNP - A General Monte Carlo N-ParticleTransportCode, Version 5, Los Alamos National Laboratory, Los Alamos, New Mexico, USA, April 2003.

[8] JEFF3.3 nuclear data library, http://www.oecdnea.org/dbdata/jeff/jeff33/\#neutron.

[9] M.J. Norgett, M.T. Robinson, I.M. Torrens, A proposed method of calculating displacement dose rates, Nuclear Engineering and Design 33 (1975) 50-54, North-Holland publishing Company.

[10] www.spaceclaim.com.

[11] Y. Wu, FDS Team, CAD-based interface programs for fusion neutron transport simulation, Fus. Eng. Des., 84, (2009), $1987-1992$.
[12] P. Frosi et al., LOADS SPECIFICATION (LS) FOR DIVERTOR CASSETTE 2018 (INCL. NEUTRONICS, EM ANALYSIS) Report of DIV-1-T005-D005 WP 2018 (2NLWLE V1.0).

[13] C. Bachmann et al., 'Overview over DEMO design integration challenges and their impact on component design concepts', Fus. Eng. Des., 136, (2018), 87-95.

[14] F. Moro et al., 'Nuclear analysis of the Water Cooled Lithium Lead DEMO reactor', this conference.

[15] U. Fischer et al., Technical Report Design Criteria, Codes and Standards ENS-1.1.5.0-NT-00-R10: Material compositions for PPPT neutronics and activation analyses, EFDA_D_2MM3A6v1.1 (2018).

[16] G. Ponti et al., "The role of medium size facilities in the HPC ecosystem: the case of the new CRESCO4 cluster integrated in the ENEAGRID infrastructure", Proceedings of the 2014 International Conference on High Performance Computing and Simulation, HPCS 2014, art. no. 6903807, 1030-1033.

[17] W. Timmis, Material Assessment Report on the Use of Copper Alloys in DEMO EFDA Report WP12-MAT02-M03, 2013.

[18] P. Fenici et al., Effect of fast-neutron irradiation on tensile properties of precipitation-hardened $\mathrm{Cu}-\mathrm{Cr}-\mathrm{Zr}$ alloy J. Nucl. Mater. 212-215 (1994) 399-403.

[19] J.H. You et al., Progress in the initial design activities for the European DEMO divertor: Subproject "Cassette", Fusion Engineering and Design 124 (2017) 364-370. 